\title{
Characterization of primary ovarian cancer cells in different culture systems
}

\author{
TE LIU ${ }^{1 *}$, WEIWEI CHENG ${ }^{1 *}$, DONGMEI LAI ${ }^{1}$, YONG HUANG ${ }^{1}$ and LIHE GUO ${ }^{2}$ \\ ${ }^{1}$ The International Peace Maternity and Child Health Hospital, Shanghai Jiaotong University, \\ Shanghai 200030; ${ }^{2}$ Hehong Biotechnology, Ltd., Shanghai 201203, P.R. China
}

Received November 16, 2009; Accepted December 21, 2009

DOI: $10.3892 /$ or_00000761

\begin{abstract}
The concept of cancer stem cells (CSCs) provides a new paradigm for understanding cancer biology. Here we report how culture conditions affect the characteristics of primary ovarian cancer cells. Cancer cells disaggregated from ovarian serous adenocarcinoma and maintained in serumfree system culture formed sphere cells that exhibited several properties expected for CSCs. These include self-renewal, overexpression of stemness genes as detected by QPCR analysis, greater tumorigenicity and enhanced drug resistance. The serum-free culture system enriched the percentage of

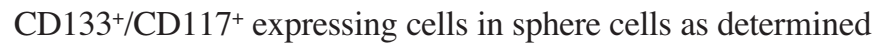
by flow cytometric analysis, immunostaining and Western blot analysis. A cDNA microarray showed that there were 2111 genes exhibiting more than a 2 -fold difference in expression. Subsequent ontological analysis revealed that a large proportion of the classified genes were related to cell communication, cell-cell adhesion, cellular development and extracellular matrix. We suggest that the sphere cell subpopulation may be a more reliable model than differentiated cells grown in the presence of serum for understanding the biology of primary ovarian cancer.
\end{abstract}

\section{Introduction}

Increasing evidence suggests that only a minority of cancer cells with stem cell properties, cancer stem cells (CSCs), are responsible for maintenance and growth of tumors (1-3). Recent advances in stem cell biology enable the identification of such CSCs in ovarian cancers (4-6). The isolation of CSC cells has provided a rationale for re-evaluating our

Correspondence to: Dr Dongmei Lai, The International Peace Maternity and Child Health Hospital, Shanghai Jiaotong University, 910 Hengshan Road, Shanghai 200030, P.R. China

E-mail: laidongmei@hotmail.com

${ }^{*}$ Contributed equally

Key words: primary ovarian cancer, sphere cells, cancer stem cell, chemoresistance, serum-free culture, cDNA microarray inability to completely eradicate ovarian cancers; although chemotherapy can shrink tumors, a small population of more resistant CSC cells may escape current regimens and seed tumor regeneration.

Recent reports have demonstrated that CSCs from epithelial organs can be expanded as sphere-like cellular aggregates in serum-free medium (SFM) containing epidermal growth factor (EGF) and basic fibroblast growth factor (bFGF) (7-9), a method derived from the culturing of neural stem/progenitor cells (NSCs) $(9,10)$. All established laboratory cell lines (like most other cancer cell lines) are grown in media containing serum, whereas NSCs are grown in serum-free media, since serum causes irreversible differentiation of NSCs $(11,12)$. In recent years, CSCs have also been isolated from solid tumors using serum-free stem cell selective culture conditions (13-19).

A major factor in the in vitro propagation of cancer cells is the presence or absence of serum. We have explored how these two different culture conditions, DMEM containing $10 \%$ fetal bovine serum or serum-free media supplemented with growth factors, affect the growth of primary ovarian cancer cells. Single cell suspensions of freshly resected and dissociated ovarian cancer tissues from the same tumor were cultured under conditions optimal for propagation and nondifferentiation of CSCs, or differentiation conditions in the presence of serum. The resulting cell populations differed in their morphology, ability to form tumors in the mouse xenograft model, and sensitivity to chemotherapeutic agents. We conclude that cancer cells propagated in the presence of serum are an inadequate model for studying tumorigenesis and cells propagated under alternative culture conditions may more closely resemble the in vivo situation.

\section{Materials and methods}

Tissue collection and grading. The present study was approved by the institutional review boards at Shanghai Jiaotong University and informed consent was obtained from three patients. The three patients' tumors (T1-T3) used in this study were categorized as stage III, grade 2-3 serous adenocarcinoma according to the International Federation of Gynecology and Obstetrics (FIGO) classification.

Cell culture. Tissue was washed, minced, suspended in McCoy's medium (Sigma), and mixed with $1 \%$ collagenase 
Table I. PCR primer sequences.

\begin{tabular}{ll}
\hline Gene product & Forward (F) and reverse (R) primers $\left(5^{\prime} \rightarrow 3^{\prime}\right)$ \\
\hline Nanog & F: GGGCCTGAAGAAAACTATCCATCC $(\mathrm{bp})$ \\
& R: TGCTATTCTTCGGCCAGTTGTTT \\
OCT-4 & F: GGCCCGAAAGAGAAAGCGAACC \\
& R: ACCCAGCAGCCTCAAAATCCTCTC \\
Sox-2 & F: GCGCGGGCGTGAACCAG \\
& R: CGGCGCCGGGGAGATACA \\
Nestin & F: CAGCTGGCGCACCTCAAGATG \\
& R: AGGGAAGTTGGGCTCAGGACTGG \\
CD133 & F: TGGATGCAGAACTTGACAACGT \\
& R: ATACCTGCTACGACAGTCGTGGT \\
CD117 & F: FCAAGGAAGGTTTCCGAATGC \\
& R: CCCAGCAGGTCTTCATGATGT \\
ABCG2 & F: CTGAGATCCTGAGCCTTTGG \\
& R: AAGCCATTGGTGTTCCTTG \\
& F: CGTTGATTAAGTCCCTGCCCTT \\
& R: TCAAGTTCGACCGTCTTCTCAG \\
\hline
\end{tabular}

and $1 \%$ hyaluronidase (Invitrogen), followed by overnight incubation $\left(37^{\circ} \mathrm{C}, 5 \% \mathrm{CO}_{2}\right)$. Enzymatically disaggregated suspensions were filtered $(70-\mu \mathrm{m}$ cell strainer) and washed twice with PBS. The resulting single tumor cells were separated in a gradient of Percoll Plus (the density of top band was $45 \%$ and the bottom layer was 20\%) (GE Healthcare Life Science). The tumor cells were mostly in the middle band and were separately maintained in two different culture systems. Some were suspended in the serum-free DMEM/ F12 supplemented with $5 \mu \mathrm{g} / \mathrm{ml}$ insulin (Sigma), $10 \mathrm{ng} / \mathrm{ml}$ human recombinant epidermal growth factor (EGF; Invitrogen), $10 \mathrm{ng} / \mathrm{ml}$ basic fibroblast growth factor (bFGF, Invitrogen), $12 \mathrm{ng} / \mathrm{ml}$ leukemia inhibitory factor (LIF, Gibco) and $0.3 \%$ bovine serum albumin (BSA; Sigma) at a density of 100000 cells per ml. These cells formed non-adherent spheres. The other tumor cells were maintained under standard conditions (DMEM/F12 supplemented with $10 \%$ fetal bovine serum (FBS) without growth factors) and formed attached differentiated cells. All cells were incubated at $37^{\circ} \mathrm{C}$ in a humidified atmosphere containing $5 \% \mathrm{CO}_{2}$.

Drug resistance assessment. Sphere cells $\left(2 \times 10^{4}\right)$ or differentiated cells (under differentiating conditions) were plated in 96-well microtiter plates in culture medium containing cisplatin $(40 \mu \mathrm{mol} / \mathrm{l})$ and pacilitaxel $(10 \mu \mathrm{mol} / \mathrm{l})$ (Sigma) for $48 \mathrm{~h}$ (6). Cultures were set up in triplicate. Proliferation condition was monitored by MTT assay and the OD reading at $490 \mathrm{~nm}$. The percentage inhibition rate was determined as follows: 1 - (sample OD490-blank control OD490)/(control OD490-blank control OD490).

RNA extraction and real-time QPCR analysis. Total RNA was extracted form sphere cells and differentiated cells using the RNeasy mini kit (Qiagen). Five hundred nanograms of total RNA from each sample were utilized for reverse transcription (RT) using the iScript cDNA synthesis kit (Bio-Rad). Real-time PCR was carried out on cDNA using IQ SYBR Green (Bio-Rad) with Mastercycler EP realplex (Germany). All reactions were performed in a $25-\mu 1$ volume. The primers for the marker gene are shown in Table I. PCR was performed by an initial denaturation at $95^{\circ} \mathrm{C}$ for $5 \mathrm{~min}$, followed by 40 cycles for $30 \mathrm{sec}$ at $95^{\circ} \mathrm{C}, 30 \mathrm{sec}$ at $60^{\circ} \mathrm{C}$ and $30 \mathrm{sec}$ at $72^{\circ} \mathrm{C}$. PCR with water instead of the template was used as a negative control. Specificity was verified by melting curve analysis and agarose gel electrophoresis. The threshold cycle $(\mathrm{Ct})$ values of each sample were used in the post-PCR data analysis. 18s RNA was used as an internal control for mRNA level normalization.

Western blotting. The sphere cells or differentiated cells were pooled and homogenized in the sample buffer. Total proteins were measured using the BCA kit (Pierce, Gaithersburg, MD) according to the manufacturer's protocol. Twenty micrograms of protein was separated on a $12 \%$ SDS-PAGE (sodium dodecyl sulfate polyacrylamide gel electrophoresis) gel and transferred to a nitrocellulose membrane. The membrane was then incubated with the primary antibody against CD133, CD117 or B-actin (rabbit anti-human/mouse at a dilution of 1:200, Boshide, Wuhan, China; or rabbit antihuman/mouse at a 1:1000 dilution (Cell Signaling, USA) at room temperature overnight. After thorough washing, the nitrocellulose was incubated with peroxidase-linked goat anti-rabbit-IgG (1:1000, Santa Cruz Biotechnology, Santa Cruz, CA) at room temperature for $1 \mathrm{~h}$. Following careful washing, staining of the immunoreactive species was performed with a Western lightning ECL kit (Perkin-Elmer Life 
Science, USA), and spot densitometry was performed using the ChemiImager imaging system (G: BOX Syngene, Gene Co., Limited, Hong Kong). The relative expression of CD133 or CD117 was presented as the ratio to $ß$-actin in each sample.

In vivo xenograft experiments. All animal studies adhered to the protocols approved by the Institutional Animal Care and Use Committee of Shanghai Jiaotong University, Shanghai, China. The sphere cells or differentiated cells were counted, resuspended in $40 \mu 1 \mathrm{PBS}$ and injected s.c. into the two sides of the flanks of 3- to 4-week-old female severe combined immunodeficient (SCID) mice. Engrafted mice were inspected biweekly for tumor appearance by visual observation and palpation until the tumor formed. Mice were sacrificed by cervical dislocation at a tumor diameter of $1 \mathrm{~cm}$. Xenograft tumors were resected, fixed in $10 \%$ phosphate-buffered formalin, and embedded in paraffin for sectioning $(5 \mu \mathrm{m})$ on a rotary microtome, followed by slide mounting H\&E staining, and histological assessment by a pathologist for tumor type and grade.

Immunofluorescence studies. Sphere cells were cytospun onto glass slides, fixed in ice-cold $4 \%$ paraformaldehyde $\left(4^{\circ} \mathrm{C}, 10 \mathrm{~min}\right)$ and blocked (30 min with normal serum). An indirect immunofluorescent labeling technique was used to identify CD133-expressing and CD117-expressing cells using mouse anti-CD133 1:200 (Cell Signaling) and rat antiCD117 1:200 monoclonal antibodies (Boshide) in PBS with $2 \%$ normal serum $(1 \mathrm{~h}$ at room temperature). Slides were washed with PBS for $5 \mathrm{~min}$ and incubated in the dark at room temperature for $30 \mathrm{~min}$ with Rodamine-conjugated goat anti-mouse IgG (against anti-CD133, Invitrogen) and FITCconjugated chicken anti-rat IgG (against anti-CD117, Invitrogen). Positive control cells were stained for each antibody, in parallel and negative controls were performed by substituting for the primary anti-bodies with mouse nonspecific IgG. Nuclei were counter-stained with Hochest 33342. Fluorescence microscopy was performed (Nikon E800 fluorescent microscope fitted with FITC and Rodamine filters), and images were acquired digitally using MagnaFire Software (Optronics) and processed in Adobe Photoshop.

Flow cytometric analysis. The expression of a panel of CD133 and CD117 markers was evaluated on cells obtained from sphere cells or from differentiated cells. Cells $\left(1 \times 10^{6}\right)$ were suspended in 2\% BSA/PBS and labeled with antiCD133 (Cell Signaling), anti-CD117 (Boshide) and (Rodamine-labeled and FITC-labeled) secondary antibodies. Isolation of $\mathrm{CD} 133^{+}, \mathrm{CD}_{117^{+}}$or $\mathrm{CD} 133^{+} \mathrm{CD} 117^{-}$cells was performed using a FC500 flow cytometer (Beckman Coulter) and analyzed by Beckman Coulter CXP software.

cDNA microarray analysis. Total RNA was labeled using Agilent's Low RNA Input Fluorescent Linear Amplification kit. Cy3-dCTP or Cy5-dCTP was incorporated during reverse transcription of $5 \mu \mathrm{g}$ total RNA into cDNA. The cDNA probes from the sphere cells were incorporated with $\mathrm{Cy} 3$, while those from differentiated cells were incorporated with $\mathrm{Cy} 5$. Different fluorescently labeled cDNA probes were mixed in $30 \mu 1$ hybridization buffer (3X SSC, $0.2 \%$ SDS, 5 X Denhardt's solution and $25 \%$ formamide) and applied to the microarray
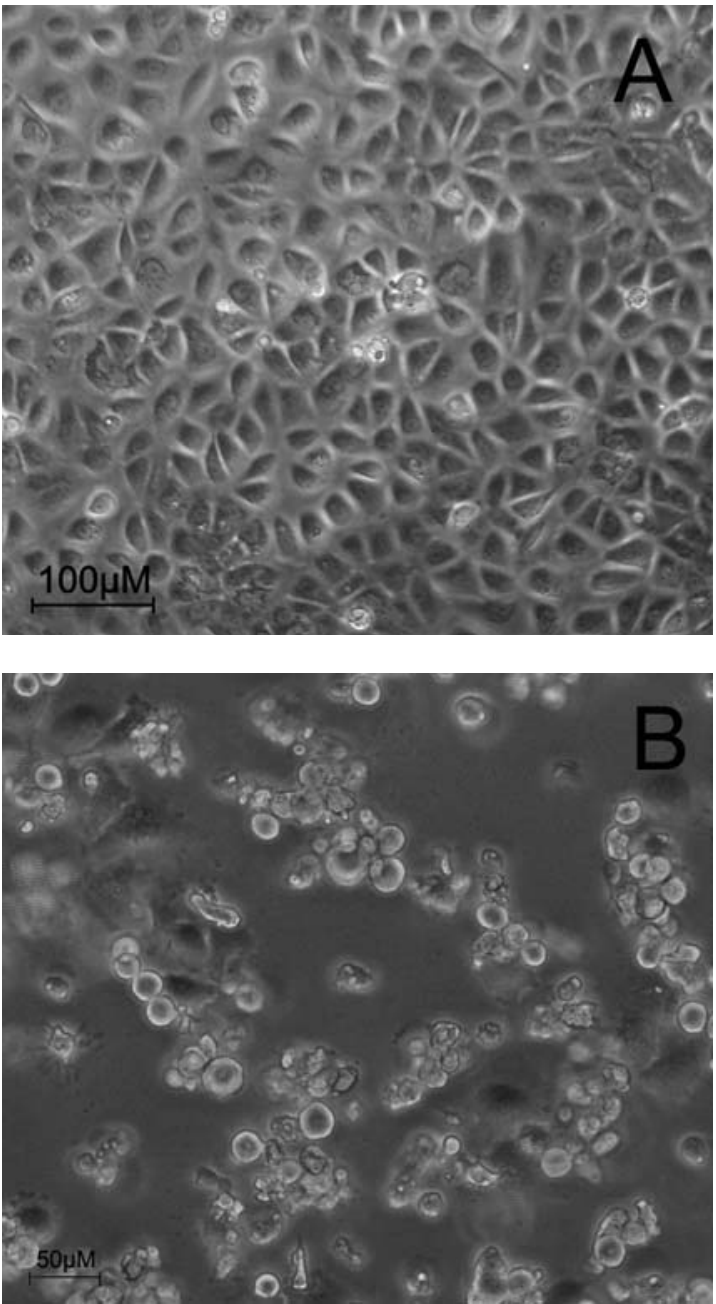

Figure 1. Phenotype of primary ovarian cancer cells (T1). (A) Ovarian cancer cells cultured under differentiation conditions. (B) Ovarian cancer cell suspensions form small, non-adherent, spheres under stem cell-selective conditions.

following incubation at $42^{\circ} \mathrm{C}$ for $16 \mathrm{~h}$. After hybridization, the slide was washed with $0.2 \% \mathrm{SDS} / 2 \mathrm{X} \mathrm{SSC}$ at $42^{\circ} \mathrm{C}$ for $5 \mathrm{~min}$, and then was washed with $0.2 \mathrm{X} \mathrm{SSC}$ at room temperature for $5 \mathrm{~min}$. The fluorescent images of the hybridized microarray were scanned with an Agilent Whole Human Genome 4x44 microarray scanner system (Santa Clara, CA, USA). Images and quantitative data of the gene-expression levels were analyzed by Agilent's Feature Extraction (FE) software, version 9.5 .

Statistical analysis. The results of the experimental data obtained from multiple experiments are reported as mean \pm $\mathrm{SD}$. The significance of differences in mean values was determined using Student's t-test, with $\mathrm{P}<0.05$ conferring statistical significance. All experiments were performed in triplicate.

\section{Results}

Sphere cell formation from primary ovarian cancer tissues under stem cell selection conditions. Primary tumor specimens obtained from three patients (FIGO stage III, grade 2-3 serous adenocarcinoma) were separately dissociated and cultured in serum-free medium supplemented with EGF, bFGF, insulin and LIF. We found that primary cancer cells 


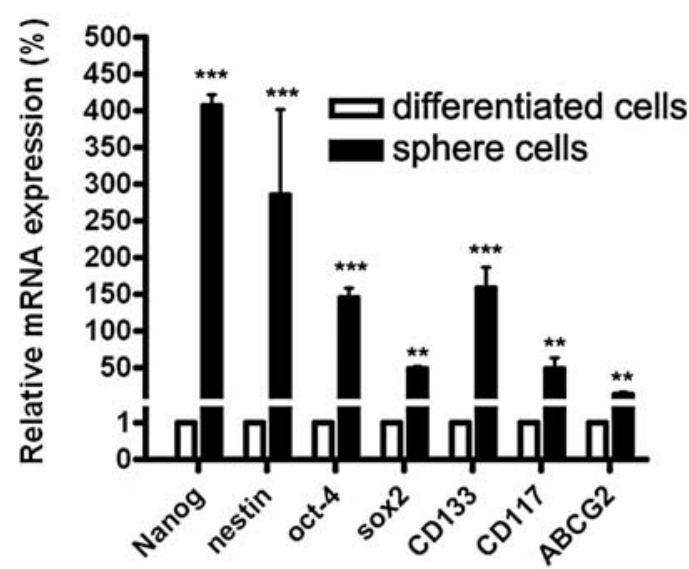

Figure 2. As shown by real-time PCR, the sphere cells, under stem cellselective conditions, overexpress stem cell marker genes compared with the differentiated cells (T1, relative to $18 \mathrm{~S}$ RNA internal control, ${ }^{* *} \mathrm{P}<0.01$, $\left.{ }^{* * *} \mathrm{P}<0.001\right)$.

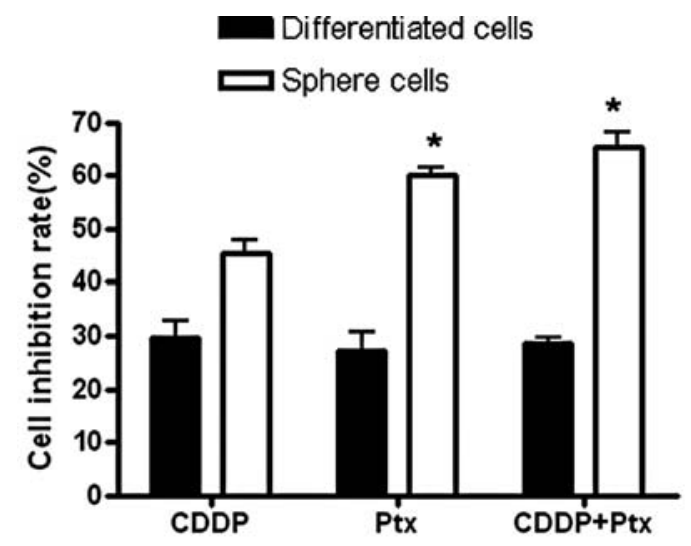

Figure 3. The sphere cells are more drug resistant compared with the differentiated cells after $48 \mathrm{~h}$ treatment with cisplatin (CDDP, $40 \mu \mathrm{mol} / \mathrm{l}$ ) and paclitaxel (Ptx, $10 \mu \mathrm{mol} / \mathrm{l})$. $\left(\mathrm{T} 1,{ }^{*} \mathrm{P}<0.05\right)$.

can form and maintain non-adherent spheres. The spheres were enzymatically dissociated to single cells, which in turn gave rise to secondary spheres. The formation of spheres could be observed in culture 7 days after plating (Fig. 1B). These cluster cells were small, non-adherent and nonsymmetric. The dissociated cancer cells were also cultured under differentiating conditions and these cells adhered to plates and formed compact clusters of relatively uniform epithelial cells (Fig. 1A). Sphere cells reproducibly grow faster than the cells grown under differentiating condition after 5 days of culturing (data not shown).

The primary ovarian cancer sphere cells have characteristics and drug resistance properties expected for stem cells. The stem/progenitor cell phenotype of the sphere cells was further confirmed by the expression of putative stem cell markers. Quantitive real-time PCR showed the expression of Nanog, Oct4, sox2, nestin, CD133, CD117 and ABCG2 in sphere cells to be higher than in differentiated cells (Fig. 2) $(\mathrm{P}<0.01)$. To examine whether self-renewing sphere cells
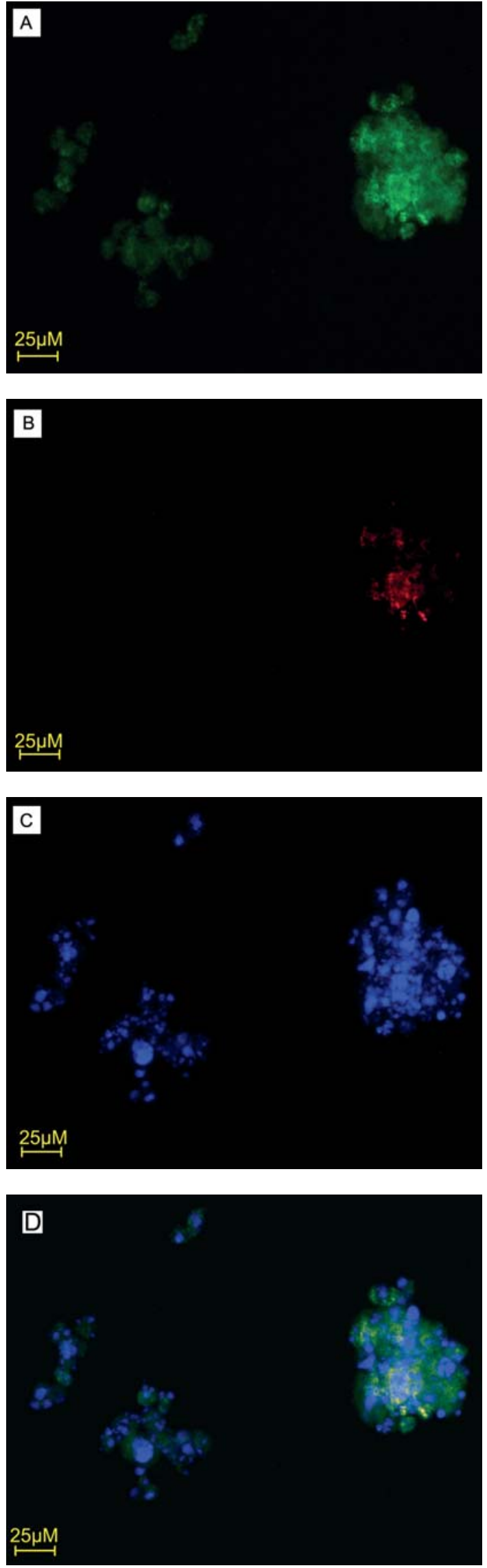

Figure 4. Representative double staining for CD133 and CD117 in the sphere cells by immunofluorescence (T1). (A) Immunofluorescence staining with anti-CD133 monoclonal antibodies (FITC-conjugated secondary antibody, green). (B) Immunofluorescence staining with anti-CD117 monoclonal antibodies (rodamine-conjugated secondary antibody, red). (C) Nuclear staining with Hochest33342 (blue). (D) Merged image of (A), (B) and (C). 


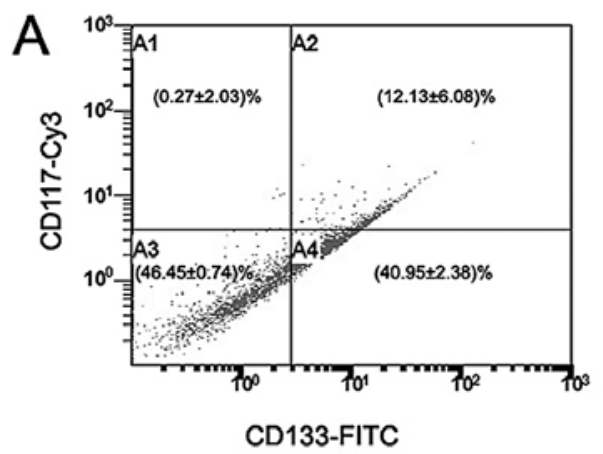

C

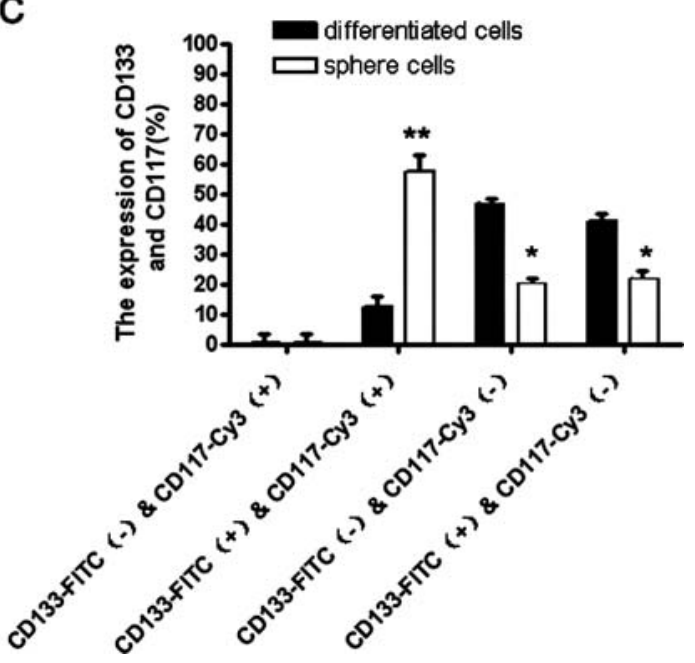

Figure 5. Flow cytometry analysis (T1). (A) CD133/CD117 expression in differentiated cells. (B) CD133/CD117 expression in sphere cells. (C) The

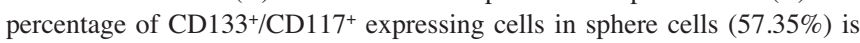
much higher than that in differentiated cells $(12.13 \%)\left({ }^{*} \mathrm{P}<0.05,{ }^{* *} \mathrm{P}<0.01\right)$.

(which have higher expression of ABCG2) possess a hypothesized cancer stem cell chemoresistant phenotype, we assessed the sensitivity of the sphere-forming cells to cisplatin and paclitaxel under stem cell selective versus differentiating conditions. After $48 \mathrm{~h}$ culture in the presence of the drugs, we found the differentiated cells to be sensitive to both cisplatin $(40.0 \mu \mathrm{mol} / \mathrm{l})$ and paclitaxel $(10.0 \mu \mathrm{mol} / \mathrm{l})$ and the inhibition reached 50-70\%. In contrast the sphere cells grown under stem cell conditions exhibited higher resistance with inhibition of $20-30 \%$ (Fig. 3) $(\mathrm{P}<0.01)$.

The presence of CD133 and CD117 proteins on the cell surface in primary ovarian cancer sphere cells. To determine whether CD133 and CD117 are present on the cell surface in primary ovarian cancer sphere cells, we carried out immunofluorescence, flow cytometric analysis and Western blot analysis. The sphere cells stained for both CD133 and CD117 (Fig. 4); in contrast, CD133 or CD117 staining in the differentiated cells was lower or undetectable (data not shown). Consistent with the immunofluorescence analysis, the flow cytometric experiments showed the percentage of CD133+/CD $117^{+}$ expressing cells in the sphere cells is 57.35 , which is much higher than that in differentiated cells $(12.13 \%)(\mathrm{P}<0.01$, Fig. 5). Furthermore, the Western blot analysis showed the expression levels of CD133 and CD117 in the sphere cells is significantly higher than that in differentiated cells $(\mathrm{P}<0.05$,

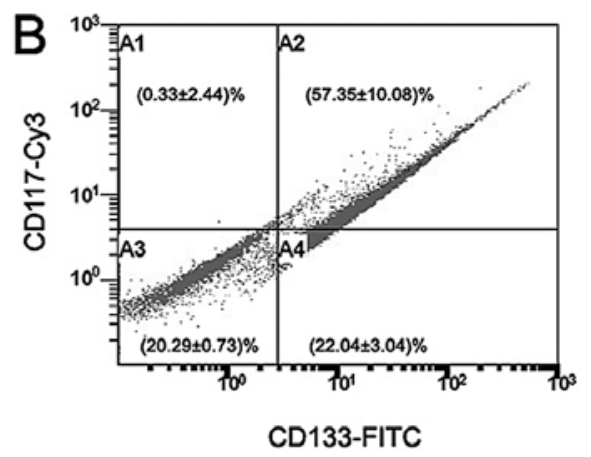

A

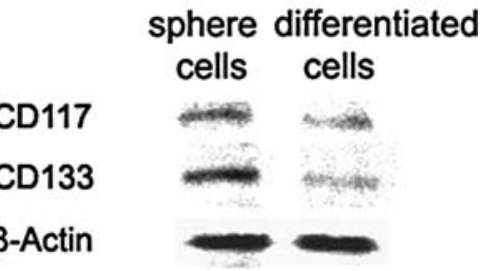

B

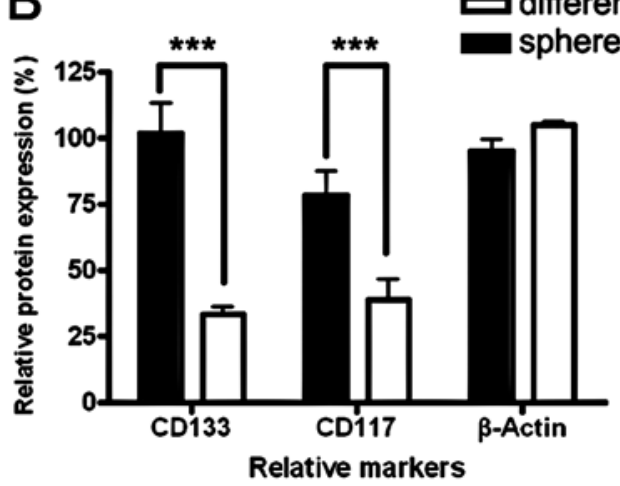

Figure 6. CD133 and CD117 expression in sphere cells and differentiated cells by Western blot analysis (T1). (A) The expression of CD133 and CD117 in sphere cells and differentiated cells, $B$-actin is an internal control. (B) The relative expression of $\mathrm{CD} 133$ and $\mathrm{CD} 117$ is presented as the ratio to $\beta$-actin in each sample, the expression levels of CD133 and CD117 in the sphere cells is significantly higher than that in differentiated cells $\left({ }^{* * *} \mathrm{P}<0.001\right)$.

Fig. 6). These results show that $\mathrm{CD} 133^{+}$and $\mathrm{CD} 117^{+}$cells are enriched under serum-free stem cell selective conditions.

The primary ovarian cancer sphere cells are highly tumorigenic and can propagate their original tumor phenotype. To investigate the tumorigenicity of primary ovarian cancer sphere cells, 10-500 disaggregated sphere cells from three patients were injected s.c. into the two sides of the flanks of athymic nude mice. Sphere cells (500) formed tumors with an average of 69 days tumor latency, whereas $10^{7}$ differentiated cells were required for tumorigenicity (Table II). All subcutaneous xenograft tumors derived from sphere cells or differentiated cells were categorized as serous adenocarcinomas of grade 2-3, which was the original tumor phenotype of the ovarian cancer (data not shown).

Differences in genetic expression between the sphere cells and differentiated cells. To further investigate the differences 
Table II. In vivo tumorigenicity of primary ovarian cancer cells (T1-T3).

\begin{tabular}{lcccc}
\hline Cell type & Cell no. per injection & Injection site (left and right) & Tumor formation & Latency (d) \\
\hline Sphere cells & $100-400$ & s.c. & $0 / 12$ & - \\
Sphere cells & 500 & s.c. & $3 / 12$ & $62-76$ \\
Differentiated cells & 1000000 & s.c. & $3 / 3$ & $90-105$ \\
Differentiated cells & 100000 & s.c. & $0 / 6$ & - \\
Differentiated cells & 10000 & s.c. & $0 / 6$ & - \\
\hline
\end{tabular}

between the sphere cells and differentiated cells, the gene expression profile was analyzed with Agilen human cDNA microarray analysis. Genes (2111) displayed more than a 2-fold difference in expression, with 1282 genes being upregulated and 829 genes down-regulated. Approximately 36 (genes) of the differentially expressed genes exhibited a $>10$-fold change, of which 21 genes were decreased and 15 genes were increased. Some of the identified genes were then assigned to a functional class using the genespring 10 (Algorithm) gene ontology annotation tool (Table III). Subsequent ontological analysis revealed that a large proportion of the classified genes were related to cell communication, cell-cell adhesion, cellular development and extracellular matrix.

\section{Discussion}

Ovarian cancer is one of the leading causes of death among gynecologic malignancies. Optimal cytoreductive surgery followed by systemic chemotherapy with paclitaxel and cisplatin is the current standard therapy for metastatic ovarian cancer at diagnosis, with a reported response rate of over $70 \%$. However, the overall 5-year survival rate is only 15 $30 \%(20,21)$. One of the most important causes of failure in ovarian cancer treatment is the development of resistance to paclitaxel and platinum-based chemotherapy (22). One emerging model for the development of drug-resistant tumors invokes a pool of self-renewing malignant progenitors known as cancer stem cells (CSCs) or cancer-initiating cells (CIC). According to the CSC hypothesis, CSCs are inherently resistant to chemotherapy because of their stem cell properties, mainly their quiescence and the expression of drug membrane transporters (e.g., ABCG2). Therefore, CSCs may survive therapy and regenerate the tumor $(23,24)$.

However, the true relatedness of putative ovarian cancer stem cells to differentiated cancer cells and their exact relevance to our current in vitro and in vivo models of cancer, as well as to primary human tumors in situ, remain unclear. Using a serum-free culture system supplemented with EGF, bFGF, LIF and insulin, we found that the primary cancer cells can form and maintain sphere cells. These sphere cells possess several intrinsic properties of stem cells: self-renewal (the sphere can produce consistently large numbers of new sphere cells), and overexpression of some stemness genes, such as Nanog, Oct4, sox2, nestin. These sphere cells overexpressed ABCG2 and were more resistant to cisplatin and paclitaxel than the differentiated cells. ABCG2 is a half transporter that appears to have a protective role in a variety of stem cells to maintain progenitor cells in an undifferentiated state (25), and is often referred to as a stem cell marker. Resistance to toxic agents is one of the most important biological characteristics of cancer stem cells and we think ABCG2 may serve as the primary line of defense against the cytotoxic effects of drug in these sphere cells. Furthermore, the sphere cells are more tumorigenic than the differentiated cancer cells. Only 500 sphere-forming cells resulted in subcutaneous xenograft tumors, while $10^{7}$ differentiated cells were required to form tumors. All of these xenografts were categorized as serous adenocarcinomas of grade 3, which was the original tumor phenotype of the ovarian cancer.

Stem cell surface antigens CD133 and CD117 were also identified in the sphere cells or the differentiated cancer cells. The CD133 antigen, a 120-kDa membrane glycoprotein coded by the CD133 gene (Prom-1 gene), was first detected in $\mathrm{CD} 34^{+}$hematopoietic stem cells $(26)$. The $\mathrm{CD} 133^{+}$cells were identified as CSCs in brain and prostate cancer $(13,14)$ as well as in colon and hepatocellular carcinomas $(27,28)$. In ovarian cancer, Szotek et al reported that both 'side population' and 'non-side population' of genetically engineered mouse ovarian cancer cells (MOVCAR 7 and 4306) do not express the CD133 antigen (5). Similarly, Olempska et al failed to detect CD133 in human SKOV3 ovarian cancer cells (29). While Ferrandina et al (30) reported the presence of CD133-1- and CD133-2-expressing cells in a large series of ovarian tissues, they found that $\mathrm{CD} 133^{+}$ovarian tumor cells exhibited higher clonogenic efficiency and more extensive proliferative potential compared to CD133- cells. The stem cell factor c-Kit (CD117) encodes a transmembrane thyrosine kinase growth factor receptor. c-Kit expression was reported in both human and mouse undifferentiated embryonic stem cells with a role in maintaining their undifferentiated state and correlated with functional measures of their pluripotency $(31,32)$, c-Kit is also a proto-oncogene. Its expression was detected in several hematological malignancies and solid tumors, ranging from 2.3 to $100 \%$ in clinical samples from patients. In ovarian serous carcinoma, c-Kit was only expressed in high-grade poorly differentiated and was absent in lowgrade well-differentiated tumors, suggesting a correlation with malignant progression (33). Similarly, in the invasive ductal carcinoma type of breast cancer, c-Kit overexpression was almost exclusively in the undifferentiated tumors with 'stem cell-like' features (34). Our results showed that both the sphere cells and the differentiated cancer cells expressed CD133 and CD117 at the mRNA level by quantitative real- 
Table III. Ontological sorting of some differentially expressed genes in primary ovarian sphere cells and differentiated cells (T1-T3).

\begin{tabular}{|c|c|c|}
\hline Name & $\begin{array}{c}\text { Ratio sphere/ } \\
\text { differentiated cells }\end{array}$ & $\begin{array}{c}\mathrm{GO} \\
\text { accession }\end{array}$ \\
\hline \multicolumn{3}{|c|}{ Cell communication } \\
\hline KCNIP3 & 6.9054 & NM_013434 \\
\hline DGKG & 4.8231 & NM_001346 \\
\hline ARHGDIB & 3.8032 & NM_001175 \\
\hline SNX21 & 3.5564 & NM_033421 \\
\hline PTPRG & 2.8631 & ВC036018 \\
\hline GPR143 & 2.6201 & NM_000273 \\
\hline KLRC2 & 0.1236 & NM_002260 \\
\hline GABRA4 & 0.2147 & NM_000809 \\
\hline CCR1 & 0.3242 & NM_001295 \\
\hline BMP6 & 0.2179 & NM_001718 \\
\hline \multicolumn{3}{|c|}{ Cell-cell adhesion } \\
\hline CLDN1 & 6.2314 & NM_021101 \\
\hline ESAM & 3.1834 & NM_138961 \\
\hline FAT4 & 3.0447 & AK026709 \\
\hline PCDHB14 & 2.0156 & NM_018934 \\
\hline CD44 & 2.1155 & NM_000610 \\
\hline RGMA & 0.1125 & NM_020211 \\
\hline ICAM2 & 0.1587 & NM_000873 \\
\hline COL19A1 & 0.2114 & NM_001858 \\
\hline ROR2 & 0.3452 & NM_004560 \\
\hline \multicolumn{3}{|c|}{ Cellular development } \\
\hline ANGPT1 & 3.5647 & NM_001146 \\
\hline NOTCH1 & 2.3569 & NM_017617 \\
\hline WNT3A & 3.4565 & NM_033131 \\
\hline NRCAM & 2.8969 & NM_005010 \\
\hline CSF1 & 2.3425 & NM_172212 \\
\hline ANG & 0.4236 & NM_001145 \\
\hline BMP6 & 0.3562 & NM_001718 \\
\hline CRB1 & 0.2314 & NM_201253 \\
\hline CHRDL2 & 0.2145 & NM_015424 \\
\hline PTGS1 & 0.3567 & NM_000962 \\
\hline \multicolumn{3}{|c|}{ Extracellular matrix } \\
\hline GPC5 & 4.2875 & NM_004466 \\
\hline EMILIN2 & 3.2275 & NM_032048 \\
\hline ADAMTS5 & 3.1958 & NM_007038 \\
\hline WNT3A & 2.4565 & NM_033131 \\
\hline LAMA4 & 2.3645 & NM_002290 \\
\hline MMP12 & 2.2147 & NM_002426 \\
\hline SMOC1 & 0.2389 & NM_001034852 \\
\hline COL9A3 & 0.2854 & NM_001853 \\
\hline ANG & 0.3369 & NM_001145 \\
\hline VTN & 0.1032 & NM_000638 \\
\hline
\end{tabular}

time PCR and at the protein level by Western blot and immunofluorescence, while CD133 and CD117 expression levels in the sphere cells are higher statistically than that in the differentiated cancer cells. Our results also showed the percentage of $\mathrm{CD} 113^{+} \mathrm{CD} 117^{+}$cells in the sphere cell population are much higher than that in differentiated cells as measured by flow cytometry analysis. Taken together, the $\mathrm{CD} 133^{+} \mathrm{CD} 117^{+}$cells were enriched under the serum-free stem cell conditions.

Efforts were focused on analyzing the altered gene profile in sphere cells and differentiated cells using cDNA microarray analysis. As a result, the analysis showed that 2111 genes had more than a 2 -fold difference in expression. Approximately 40 of the differentially expressed genes exhibited a change greater than 10-fold, of which 25 genes were decreased and 15 genes increased. Through functional clustering of the differentially expressed genes, a large proportion of the classified genes were found to be related to cell communication, cell-cell adhesion, cellular development and extracellular matrix. Several potential targets detected in this study warrant further investigation.

In summary, primary cancer cells from ovarian cancer specimens form non-adherent spheres, display remarkable stem/progenitor cell properties and have higher and more stable drug resistance characteristics, and are more tumorigenic. The gene expression profile differences between the sphere cells and differentiated cells are suggestive of multiple roles in cell growth, cell signaling and chemoresistance. As we increasingly appreciate the significant heterogeneity within specific subtypes of solid tumors such as ovarian cancer and as we develop more rationally based, molecularly targeted drugs (e.g. anti-CD133 and anti-CD117), we will move toward an era of personalized therapy for individual tumors. Such an individualized therapeutic approach will require a model system for identifying and understanding the basic genotype, gene expression profiles, and/or in vitro and in vivo biological characteristics of unique tumors from individual patients. Since the sphere cells isolated from primary ovarian cancer tissues can be frozen, stored and produce consistently large numbers of cancer stem-like cells, it may be a more reliable model system for understanding the biology of primary human tumors, for screening new therapeutic agents, and ultimately for guiding clinical personalized tumor therapy. Further studies evaluating the relatedness of these sphere cells to the differentiated cancer cells and a thorough phenotypic/genotypic characterization of these sphere cells would ultimately be required to truly understand how closely they fulfill the criteria of CSCs.

\section{Acknowledgements}

The funding for this study was provided by Science and Technology Commission of Shanghai Municipality 2007 Shanghai Pujiang project (to D.L. 07pj14090), and Science and Technology Commission of Shanghai Municipality 2009 YIXUEYINGDAO project (to D.L. 09411968300), Shanghai, China.

\section{References}

1. Reya T, Morrison SJ, Clarke MF and Weissman IL: Stem cells, cancer and cancer stem cells. Nature 414: 105-111, 2001.

2. Mackenzie IC: Stem cell properties and epithelial malignancies. Eur J Cancer 42: 1204-1212, 2006. 
3. Mimeault M, Hauke R, Mehta PP and Batra SK: Recent advances in cancer stem/progenitor cell research: therapeutic implications for overcoming resistance to the most aggressive cancers. J Cell Mol Med 11: 981-1011, 2007.

4. Bapat SA, Mali AM, Koppikar CB and Kurrey NK: Stem and progenitor-like cells contribute to the aggressive behavior of human epithelial ovarian cancer. Cancer Res 65: 3025-3029, 2005.

5. Szotek PP, Pieretti-Vanmarcke R, Masiakos PT, et al: Ovarian cancer side population defines cells with stem cell-like characteristics and mullerian inhibiting substance responsiveness. Proc Natl Acad Sci USA 103: 11154-11159, 2006.

6. Zhang S, Balch C, Chan MW, et al: Identification and characterization of ovarian cancer-initiating cells from primary human tumors. Cancer Res 68: 4311-4320, 2008.

7. Ricci-Vitiani L, Lombardi DG, Pilozzi E, et al: Identification and expansion of human colon-cancer-initiating cells. Nature 445: 111-115, 2007

8. Dontu G, Abdallah WM, Foley JM, et al: In vitro propagation and transcriptional profiling of human mammary stem/progenitor cells. Genes Dev 17: 1253-1270, 2003.

9. Uchida N, Buck DW, He D, et al: Direct isolation of central nervous system stem cells. Proc Natl Acad Sci USA 97: $14720-14725,2000$

10. Kondo T, Setoguchi T and Taga T: Persistence of a small subpopulation of cancer stem-like cells in the C6 glioma cell line. Proc Natl Acad Sci USA 101: 781-786, 2004.

11. Gage FH, Ray J and Fisher LJ: Isolation, characterization and use of stem cells from the CNS. Annu Rev Neurosci 18: 159-192, 1995.

12. McKay R: Stem cells in the central nervous system. Science 276: 66-71, 1997.

13. Singh SK, Hawkins C, Clarke ID, et al: Identification of human brain tumour initiating cells. Nature 432: 396-401, 2004.

14. Collins AT, Berry PA, Hyde C, et al: Maitland, prospective identification of tumorigenic prostate cancer stem cells. Cancer Res 65: 10946-10951, 2005.

15. Dalerba P, Dylla SJ, Park IK, et al: Phenotypic characterization of human colorectal cancer stem cells. Proc Natl Acad Sci USA 104: 10158-10163, 2007.

16. Kim CF, Jackson EL, Woolfenden AE, et al: Identification of bronchioalveolar stem cells in normal lung and lung cancer. Cell 121: 823-835, 2005.

17. Li C, Heidt DG, Dalerba P, et al: Identification of pancreatic cancer stem cells. Cancer Res 67: 1030-1037, 2007.

18. Galli R, Binda E, Orfanelli U, et al: Isolation and characterization of tumorigenic, stem-like neural precursors from human glioblastoma. Cancer Res 64: 7011-7021, 2004.
19. Lee J, Kotliarova S, Kotliarov Y, et al: Tumor stem cells derived from glioblastomas cultured in bFGF and EGF more closely mirror the phenotype and genotype of primary tumors than do serum-cultured cell lines. Cancer Cell 9: 391-403, 2006.

20. Cannistra SA: Cancer of the ovary. N Engl J Med 351: 2519-2529, 2004.

21. Ozols RF: Treatment goals in ovarian cancer. Int J Gynecol Cancer 15: 3-11, 2005.

22. Thigpen JT, Aghajanian CA, Alberts DS, et al: Role of pegylated liposomal doxorubicin in ovarian cancer. Gynecol Oncol 96: 10-18, 2005.

23. Dean M, Fojo T and Bates S: Tumour stem cells and drug resistance. Nat Rev Cancer 5: 275-284, 2005.

24. Dalerba P, Cho RW and Clarke MF: Cancer stem cells: models and concepts. Annu Rev Med 58: 267-284, 2007.

25. Krishnamurthy P and Schuetz JD: Role of ABCG2/BCRP in biology and medicine. Ann Rev Pharmacol Toxicol 46: 381-410, 2006.

26. Bhatia M: AC133 expression in human stem cells. Leukemia 15: $1685-1688,2001$

27. O'Brien CA, Pollett A, Gallinger S and Dick JE: A human colon cancer cell capable of initiating tumour growth in immunodeficient mice. Nature 445: 106-110, 2007.

28. Suetsugu A, Nagaki M, Aoki $\mathrm{H}$, et al: Characterization of $\mathrm{CD}_{133^{+}}$hepatocellular carcinoma cells as cancer stem/ progenitor cells. Biochem Biophys Res Commun 351: 820-824, 2006.

29. Olempska M, Eisenach PA, Ammerpohl O, et al: Detection of tumor stem cell markers in pancreatic carcinoma cell lines. Hepatobiliary Pancreatic Dis Int 6: 92-97, 2007.

30. Ferrandina G, Bonanno G, Pierelli L, et al: Expression of CD133-1 and CD133-2 in ovarian cancer. Int J Gynecol Cancer 18: 506-514, 2008.

31. Bashamboo A, Taylor AH, Samuel K, et al: The survival of differentiating embryonic stem cells is dependent on the SCF-Kit pathway. J Cell Sci 119: 3039-3046, 2006.

32. Palmqvist L, Glover $\mathrm{CH}$, Hsu L, et al: Correlation of murine embryonic stem cell gene expression profiles with functional measures of pluripotency. Stem Cells 23: 663-680, 2005.

33. Schmandt RE, Broaddus R, Lu KH, et al: Expression of c-ABL, $\mathrm{c}-\mathrm{Kit}$ and platelet-derived growth factor receptor-beta in ovarian serous carcinoma and normal ovarian surface epithelium. Cancer 98: 758-764, 2003.

34. Tsuda H, Tani Y, Weisenberger J, et al: Frequent KIT and epidermal growth factor receptor overexpression in undifferentiated type breast carcinomas with 'stem cell-like' features. Cancer Sci 96: 333-339, 2005. 